\title{
Using Next-Generation Sequencing to Detect Differential Expression Genes in Bradysia odoriphaga after Exposure to Insecticides
}

\author{
Haoliang Chen ${ }^{1}$, Lulu Lin ${ }^{1}$, Farman Ali ${ }^{1,2}$, Minghui Xie ${ }^{1}$, Guangling Zhang ${ }^{1}$ and Weihua Su ${ }^{1, *}$ \\ 1 Institute of Plant Protection and Agro-Products Safety, Anhui Academy of Agricultural Sciences, \\ Hefei 230031, China; chenhaoliang2004@163.com (H.C.); linle2013@163.com (L.L.); \\ drfarman@gmail.com (F.A.); xieminghui730@163.com (M.X.); zhglnxy@126.com (G.Z.) \\ 2 Department of Agriculture, Abdul Wali Khan University, Mardan 23200, Khyber Pakhtunkhwa, Pakistan \\ * Correspondence: Suwh850@163.com; Tel.: +86-551-6516-0850
}

Received: 8 October 2017; Accepted: 15 November 2017; Published: 17 November 2017

\begin{abstract}
Bradysia odoriphaga (Diptera: Sciaridae) is the most important pest of Chinese chive. Insecticides are used widely and frequently to control B. odoriphaga in China. However, the performance of the insecticides chlorpyrifos and clothianidin in controlling the Chinese chive maggot is quite different. Using next generation sequencing technology, different expression unigenes (DEUs) in B. odoriphaga were detected after treatment with chlorpyrifos and clothianidin for 6 and $48 \mathrm{~h}$ in comparison with control. The number of DEUs ranged between 703 and 1161 after insecticide treatment. In these DEUs, 370-863 unigenes can be classified into 41-46 categories of gene ontology (GO), and 354-658 DEUs can be mapped into 987-1623 Kyoto Encyclopedia of Genes and Genomes (KEGG) pathways. The expressions of DEUs related to insecticide-metabolism-related genes were analyzed. The cytochrome P450-like unigene group was the largest group in DEUs. Most glutathione S-transferase-like unigenes were down-regulated and most sodium channel-like unigenes were up-regulated after insecticide treatment. Finally, 14 insecticide-metabolism-related unigenes were chosen to confirm the relative expression in each treatment by quantitative Real Time Polymerase Chain Reaction (qRT-PCR). The results of qRT-PCR and RNA Sequencing (RNA-Seq) are fairly well-established. Our results demonstrate that a next-generation sequencing tool facilitates the identification of insecticide-metabolism-related genes and the illustration of the insecticide mechanisms of chlorpyrifos and clothianidin.
\end{abstract}

Keywords: next-generation sequencing; Bradysia odoriphaga; differential expression genes; insecticide

\section{Introduction}

Chinese chive, Allium tuberosum Rottler ex Sprengel (Asparagales: Amaryllidaceae) is considered to be an important vegetable in north China, because it is the main stuffing for dumplings. In the production of Chinese chive, leek maggot, Bradysia odoriphaga Yang and Zhang (Diptera: Sciaridae), has always been a major impediment [1]. While the adult of B. odoriphaga looks like mosquito, the larva of $B$. odoriphaga is a maggot that feeds on the chive roots and stems, causing plants to stunt or even die. Under favorable temperatures, a female can lay about one hundred eggs on the chive stems or in the moist soil near the chive plants [2]. In north China, B. odoriphaga can cause heavy losses to the production of Chinese chive or even ruin the whole crop if not treated with insecticides [3]. Therefore, many studies have been conducted on the ecology and chemical control of B. odoriphaga [1-3]. Apart from chemicals, some other potential management techniques have also been used $[4,5]$. Chlorpyrifos, which we tested, has been widely used in Anhui province to control the Chinese chive maggot, whereas clothianidin has not been used against $B$. odoriphaga 
before. The $48 \mathrm{~h} \mathrm{LC50}$ of Chlorpyrifos is as high as $25.1 \mu \mathrm{g} / \mathrm{L}$, while that of clothianidin is merely $0.5 \mu \mathrm{g} / \mathrm{L}$. The widespread frequent application of chemical insecticides (e.g., organophosphates, and neonicotinoids) is the most prevalent management strategy used against $B$. odoriphaga when planting the Chinese chive in China. This has led to an excessive use of insecticides resulting in environmental pollution and high residues on marketed chives.

The change of detoxification gene expression plays a key role in insecticide metabolism [6]. Identifying the specific genes involved in insecticide metabolism and their genetic pathways could be beneficial for controlling B. odoriphaga. However, little research has been conducted on the insecticide metabolism of this pest; therefore, limited genomic information on this pest is available in GenBank databases $[7,8]$. Next generation sequencing (NGS) technologies have dramatically developed in the last decade, which allows us to sequence DNA and RNA much more quickly and cheaply than before, and as such has revolutionized the study of genomics and molecular biology [9-11]. RNA-Seq has been used to find insecticide-metabolism-related genes in several species, such as Cimex lectularius [12], Plutella xylostella [13], Panonychus ulmi [14] and Tetranychus cinnabarinus [15].

In this study, in order to determine insecticide resistant gene/s, we performed RNA-seq for B. odoriphaga treated with an organophosphate insecticide, chlorpyrifos, and a neonicotinoid insecticide, clothianidin, with the former being used commonly in the field to control B. odoriphaga. The differential expression unigenes (DEUs) were identified using Basic Local Alignment Search Tool (BLAST) and annotated against the National Center for Biotechnology Information (NCBI) Non-Redundant (NR) dataset. Thereafter, the DEUs were categorized by gene ontology (GO) and assigned to pathways using the Kyoto Encyclopedia of Genes and Genomes (KEGG). Finally, 14 unigenes that included five cytochrome P450 (P450)-like unigenes, five glutathione S-transferase (GST)-like unigenes, two carboxylesterase-like unigenes, and two cuticle-protein-like unigenes were used in qRT-PCR to confirm their expression under different insecticide treatments.

Determination of the genetic pathways and specific genes involved in the detoxification pathway could be beneficial for the control of $B$. odoriphaga. This will give us a choice to use alternative methods for controlling this particular pest if it develops resistance to a single specific or group of insecticides.

\section{Results}

\subsection{RNA-Seq Sequencing and Assembly}

De novo transcriptome sequencing of all stages of $B$. odoriphaga generated 34,154 unigenes, with a total length of $38,551,589 \mathrm{bp}$, a mean length of $1128 \mathrm{bp}$ and an N50 (50\% of the total assembled sequence was contained in contigs of this length or longer) of $1984 \mathrm{bp}$. A total of 20,058 unigenes were annotated against the NCBI NR protein database, 5280 in GO function categories, and 15,910 unigenes were mapped onto the canonical pathways in KEGG. Fifteen samples treated with insecticides on average generated $23.99 \mathrm{Mb}$ raw sequencing reads, of which $23.97 \mathrm{Mb}$ clean reads were obtained for each sample after filtering low quality reads. For all insecticide-treated samples, the percentage of clean reads was higher than $99.10 \%$, with an average of $99.89 \%$ (Table 1 ). There were about 1.20 billion clean nucleotides for each insecticide-treated sample. The Q20 (error probability of 0.01 ) of the clean nucleotides of all samples was more than $95.50 \%$, with an average of $98.01 \%$. The GC (guanine and cytosine) percentages for each insecticide-treated sample were between $38.76 \%$ and $41.53 \%$, averaging $40.42 \%$. The percentage of clean reads from insecticide-treated samples mapped to the reference sequence ranged between $80.97 \%$ and $86.17 \%$, with an average of $83.56 \%$ (Table 1 ). 
Table 1. Statistics of clean reads.

\begin{tabular}{ccccccc}
\hline Sample & Clean Reads & Clean Reads (\%) & Clean Nucleotides (bp) & >Q20 (\%) & GC (\%) & Total Mapped Reads (\%) \\
\hline CK1 & $24,129,440$ & 99.96 & $1.21 \times 10^{9}$ & 98.18 & 40.70 & 88.36 \\
CK2 & $24,122,549$ & 99.94 & $1.21 \times 10^{9}$ & 98.28 & 39.85 & 83.93 \\
CK3 & $24,117,751$ & 99.91 & $1.21 \times 10^{9}$ & 98.34 & 38.76 & 83.85 \\
CH_6h1 & $23,946,892$ & 99.95 & $1.20 \times 10^{9}$ & 95.52 & 40.35 & 80.97 \\
CH_6h2 & $23,953,030$ & 99.98 & $1.20 \times 10^{9}$ & 98.43 & 41.06 & 86.17 \\
CH_6h3 & $23,940,066$ & 99.92 & $1.20 \times 10^{9}$ & 98.21 & 40.62 & 83.63 \\
CH_48h1 & $23,949,969$ & 99.96 & $1.20 \times 10^{9}$ & 98.37 & 40.20 & 83.07 \\
CH_48h2 & $23,741,893$ & 99.10 & $1.19 \times 10^{9}$ & 98.18 & 40.79 & 84.57 \\
CH_48h3 & $23,944,154$ & 99.94 & $1.20 \times 10^{9}$ & 98.35 & 41.53 & 84.96 \\
CL_6h1 & $23,945,882$ & 99.95 & $1.20 \times 10^{9}$ & 98.13 & 40.16 & 80.98 \\
CL_6h2 & $23,949,511$ & 99.96 & $1.20 \times 10^{9}$ & 98.22 & 40.29 & 83.80 \\
CL_6h3 & $23,948,469$ & 99.96 & $1.20 \times 10^{9}$ & 98.01 & 40.31 & 81.25 \\
CL_48h1 & $23,919,748$ & 99.84 & $1.20 \times 10^{9}$ & 98.30 & 40.62 & 83.34 \\
CL_48h2 & $23,949,005$ & 99.96 & $1.20 \times 10^{9}$ & 97.89 & 40.33 & 81.82 \\
CL_48h3 & $23,945,861$ & 99.95 & $1.20 \times 10^{9}$ & 98.43 & 40.67 & 82.78 \\
\hline
\end{tabular}

CK: Control; CH: Chlorpyrifos; CL: Clothianidin; 6 and 48 h: treatment with Chlorpyrifos or Clothianidin after 6 or $48 \mathrm{~h}$.

\subsection{Unigene Expression Analysis for Insecticide Treatment}

Clean reads were mapped to the assembled reference sequence database of $B$. odoriphaga. The number of identified unigenes was between 29,146 and 31,467, with an average of 30,736 for all samples, and the proportion to de novo database was between $85.34 \%$ and $92.13 \%$, with an average of $89.99 \%$ (Table S1). The unigene expression of samples treated with insecticides, was compared with control based on the fragments per kilobase of transcript per million mapped reads (FPKM) value. A total of 703 to 1161, with an average of 1022 unigenes were considered as expression-changed unigenes after pesticide treatment (Figure 1). B. odoriphaga exposed to chlorpyrifos for $48 \mathrm{~h}$ had the maximum number (658) of up-regulated unigenes in comparison to the control, followed by $6 \mathrm{~h}$ exposure to clothianidin (646), while the maximum number (533) of down-regulated unigenes was observed in the treatment exposed to clothianidin for $48 \mathrm{~h}$ and the minimum number (284) was found in the treatment exposed to chlorpyrifos for $6 \mathrm{~h}$ (Figure 1). The intersection and union of the DEU heat map for the same insecticide treatment and different time duration is shown in Figure 2. The intersection of the DEU number for chlorpyrifos and clothianidin treated samples with different time durations is 371 and 681, respectively, while for union it is 1414 and 1600, respectively (Spreadsheet S1). The cluster tree indicates that the clustering of insecticide-treated samples are clearly separated from the control. Within insecticide treated samples, the insects exposed to insecticides for $6 \mathrm{~h}$ formed a separate cluster to those exposed for $48 \mathrm{~h}$, although there were two different insecticides used (Figure 3).

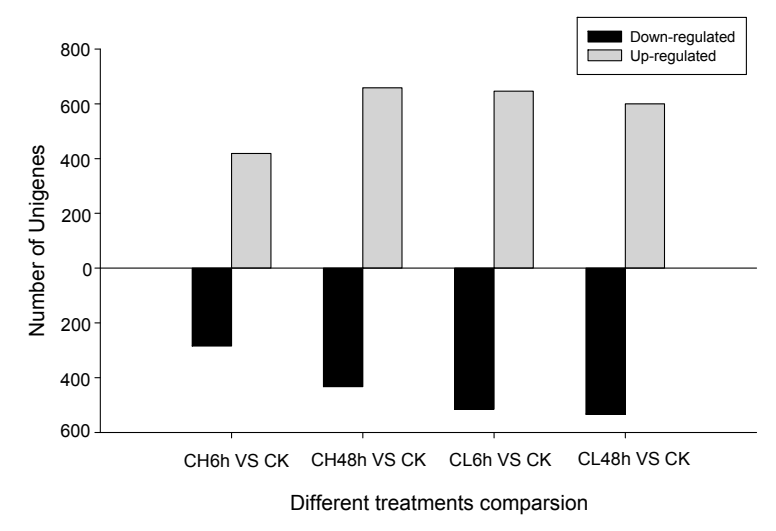

Figure 1. The number of up- and down-regulated unigenes is more than two-fold when compared between different treatments. CK: Control; $\mathrm{CH}$ : Chlorpyrifos; CL: Clothianidin; 6 and 48 h: treatment with Chlorpyrifos or Clothianidin after 6 or $48 \mathrm{~h}$. 

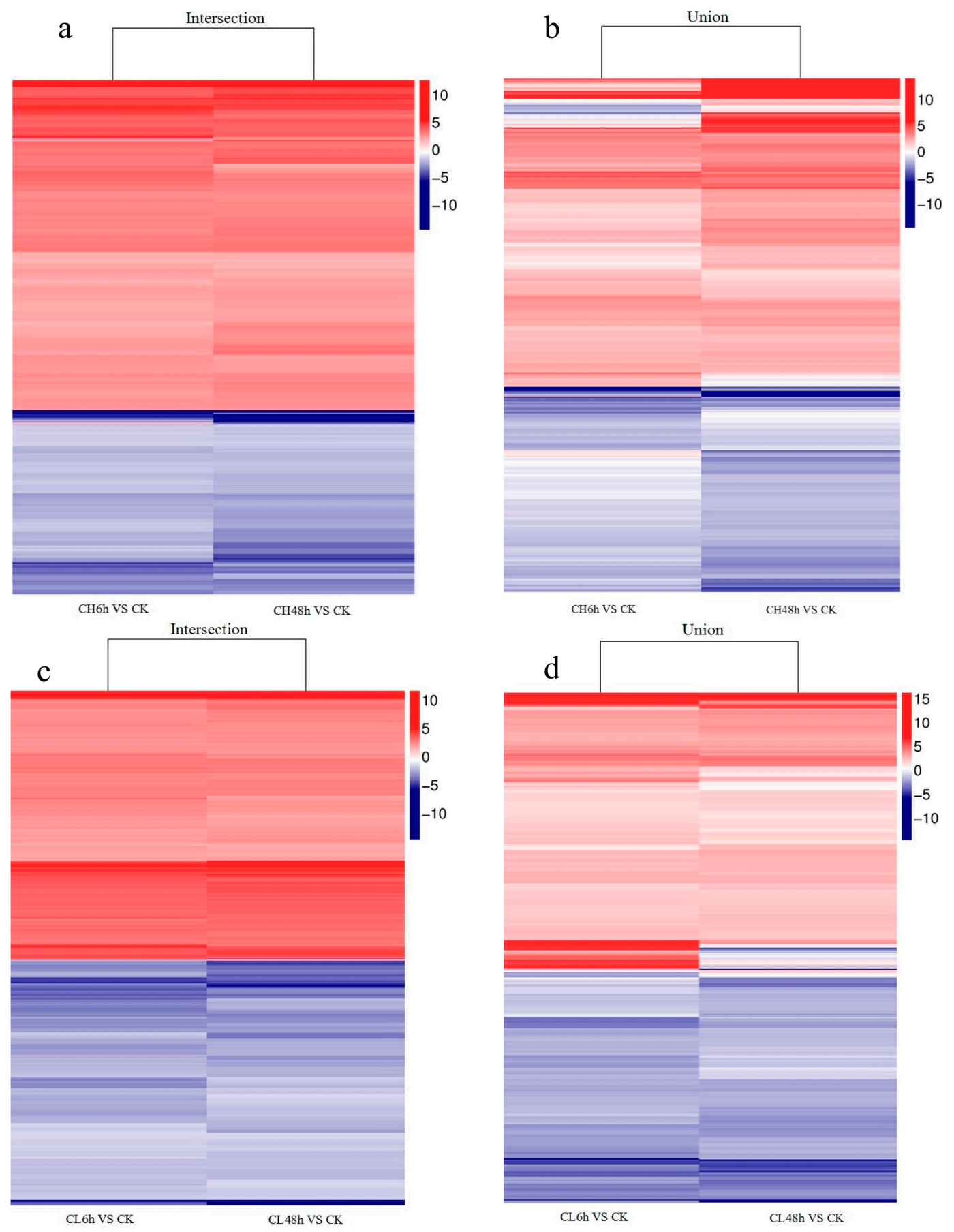

Figure 2. Heat map with dendogram for the Differential Expression Unigenes (DEUs) after treating the larvae with the same insecticide for different time durations. Gradient color barcode at the right indicates fold change of gene expression. CK: Control; CH: Chlorpyrifos; CL: Clothianidin; $6 \mathrm{~h}$ and $48 \mathrm{~h}$ : treatment with Chlorpyrifos or Clothianidin after 6 or 48 h. (a,b): DEUs for chlorpyrifos treated for 6 and $48 \mathrm{~h}$ in comparison with control, (a) is intersection and (b) is union; (c,d): DEUs for clothianidin treated for 6 and $48 \mathrm{~h}$ in comparison with control, (c) is intersection and (d) is union. 


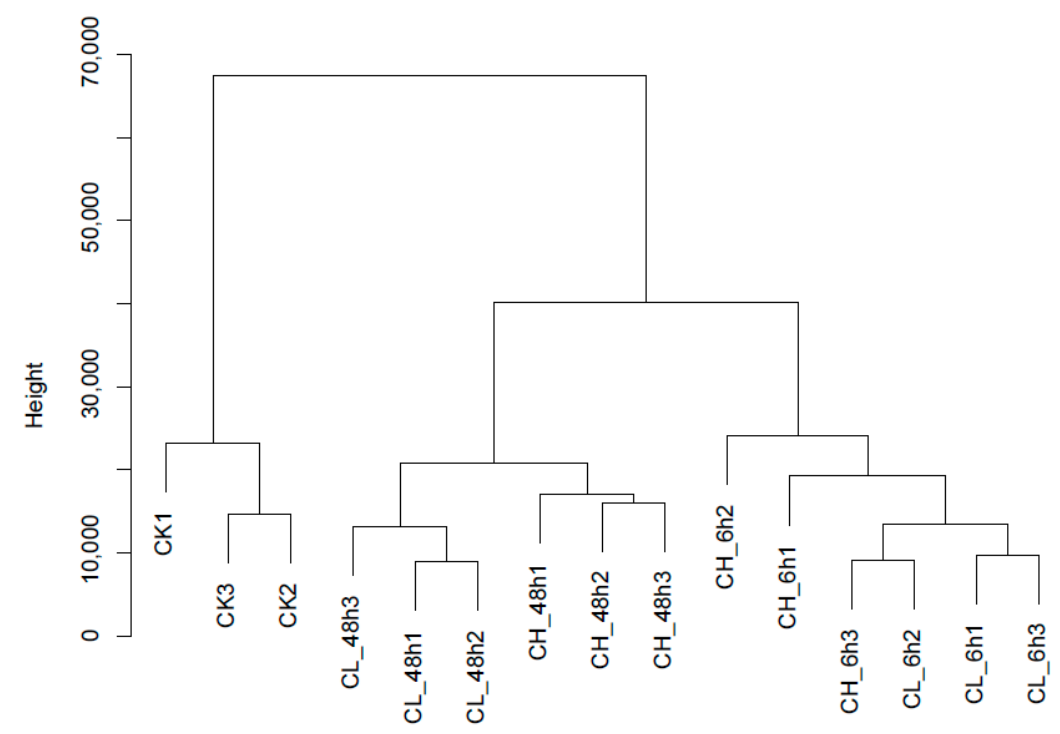

Samples

Figure 3. Cluster tree involving all samples. The distances of the expressed gene are calculated by the Euclidean method. Meanwhile, the algorithm of the sum of squares of deviations is used to calculate the distance between samples so that a cluster tree can be built. The $\mathrm{Y}$ axis means the height in the cluster tree. When samples have similar height values, they can be easily gathered. CK: Control; $\mathrm{CH}$ : Chlorpyrifos; CL: Clothianidin; 6 and 48 h: treatment with Chlorpyrifos or Clothianidin after 6 or $48 \mathrm{~h}$.

\subsection{GO Classifications and KEGG Pathway Identification of DEUs}

The identified DEUs were assigned to GO functional classification. For the DEUs, the samples treated with different insecticides for different time durations when compared with the control, generated a total of 370-863 unigenes, with an average of 581 that can be classified into $41-46$ categories of the GO functional group (Figure 4). The DEUs of clothianidin treated for $48 \mathrm{~h}$ in comparison with control had the most number of unigenes that can be classified into the GO terms, of which 863 unigenes were assigned to 46 GO classifications. In contrast, chlorpyrifos-treated samples for $6 \mathrm{~h}$ versus control had the least classification unigenes, of which 370 unigenes were assigned into 44 GO classifications (Table S2). For the DEUs, samples treated with two different insecticides for different time durations when compared with each other, generated a total of 110-225 unigenes, with an average of 149 that could be assigned into 24-35 categories of GO functional groups (Table S2). The stability of the percentages of DEUs for the three main GO categories was noted in the samples treated with insecticides versus control, rather than in insecticide versus insecticide-treated samples (Table S3). For example, the percentage of DEUs assigned to the biological process was in the range of 48.92 to 52.61 for insecticide-treated samples in comparison with control, but within insecticide-treated samples, the percentage ranged from 38.46 to 51.56 (Table S3).

To identify possible active biological pathways of DEUs, the unigenes were mapped onto the canonical pathways in KEGG. For the DEUs in the insecticide-treated sample versus control and using different exposure times, 354 to 658 DEUs, with an average of 544, could be mapped into 987 to 1623 pathways, with an average of 1399 (Figure 5). The DEUs for clothianidin-treated samples for $6 \mathrm{~h}$ in comparison with control can be mapped onto most pathways (1623), while chlorpyrifos treated samples for $6 \mathrm{~h}$ could be mapped onto the least (987) (Table S4). For the DEUs, after comparing the insecticide-treated samples with each other for different exposure times, a total of 178 to 354 DEUs, with an average of 215 , could be mapped onto $496-735$ pathways, with an average of 589 . The majority 
of the pathways (735) identified by the DEUs to be mapped after comparing the insecticide-treated samples for different time durations, were achieved with chlorpyrifos-treated samples for 6 and $48 \mathrm{~h}$, while the least number (496) was achieved with clothianidin-treated samples after 6 and 48 h exposure (Table S4). The ten most up- and down-regulated DEUs in the control samples and those treated with two pesticides for $48 \mathrm{~h}$ are listed in Tables 2 and 3. Fifteen out of the twenty most significant DEUs from the control and chlorpyrifos-treated samples can be mapped onto KEGG pathways, while this was eighteen out of twenty for clothianidin.

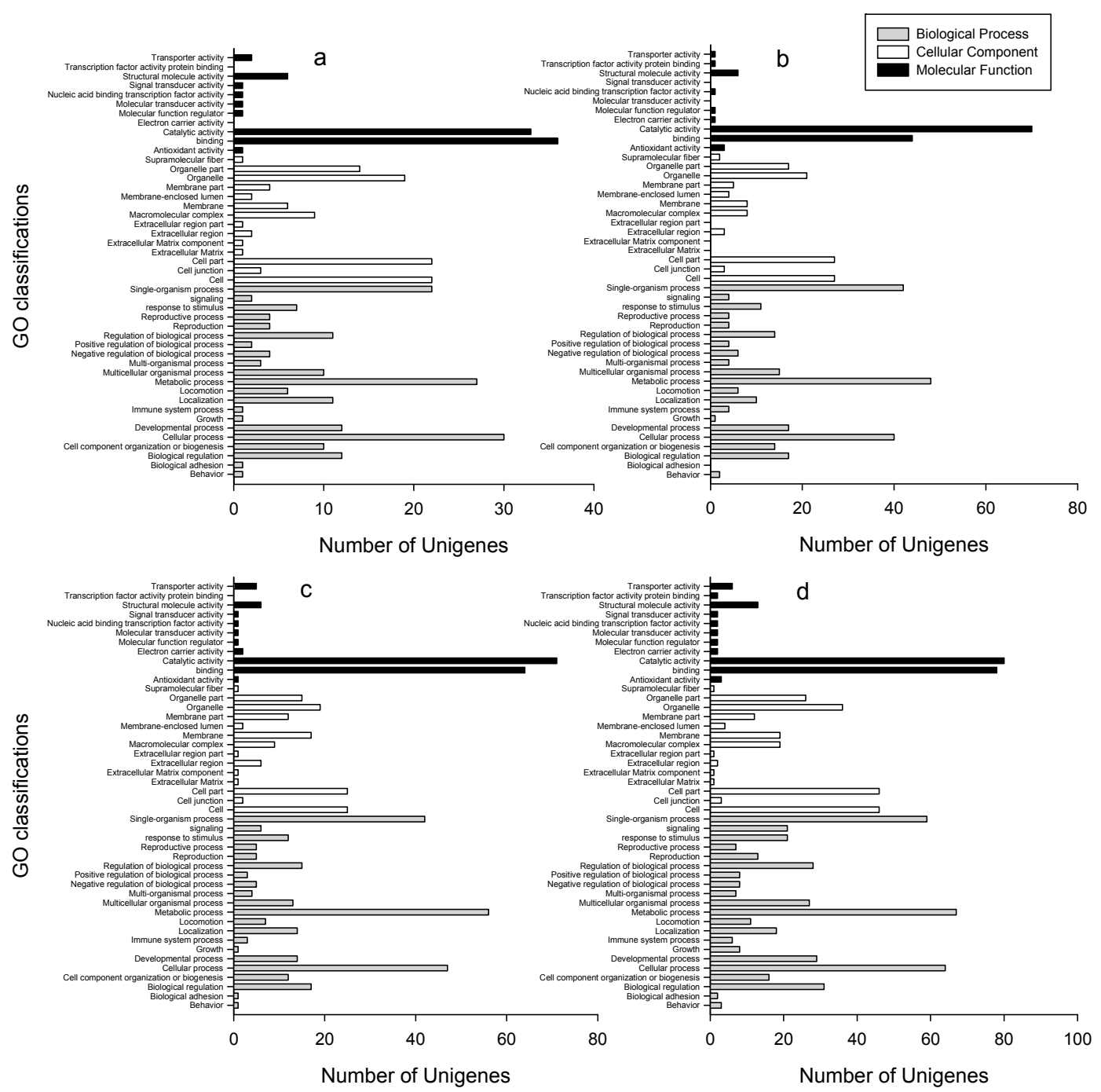

Figure 4. Number of DEUs classified into the Gene Ontology (GO) function catalogue using different insecticides and different time durations in comparison with control. (a) treatment with Chlorpyrifos for $6 \mathrm{~h}$ versus control, (b) treatment with Chlorpyrifos for $48 \mathrm{~h}$ versus control, (c) treatment with Clothianidin for $6 \mathrm{~h}$ versus control, (d) treatment with Clothianidin for $48 \mathrm{~h}$ versus control. 

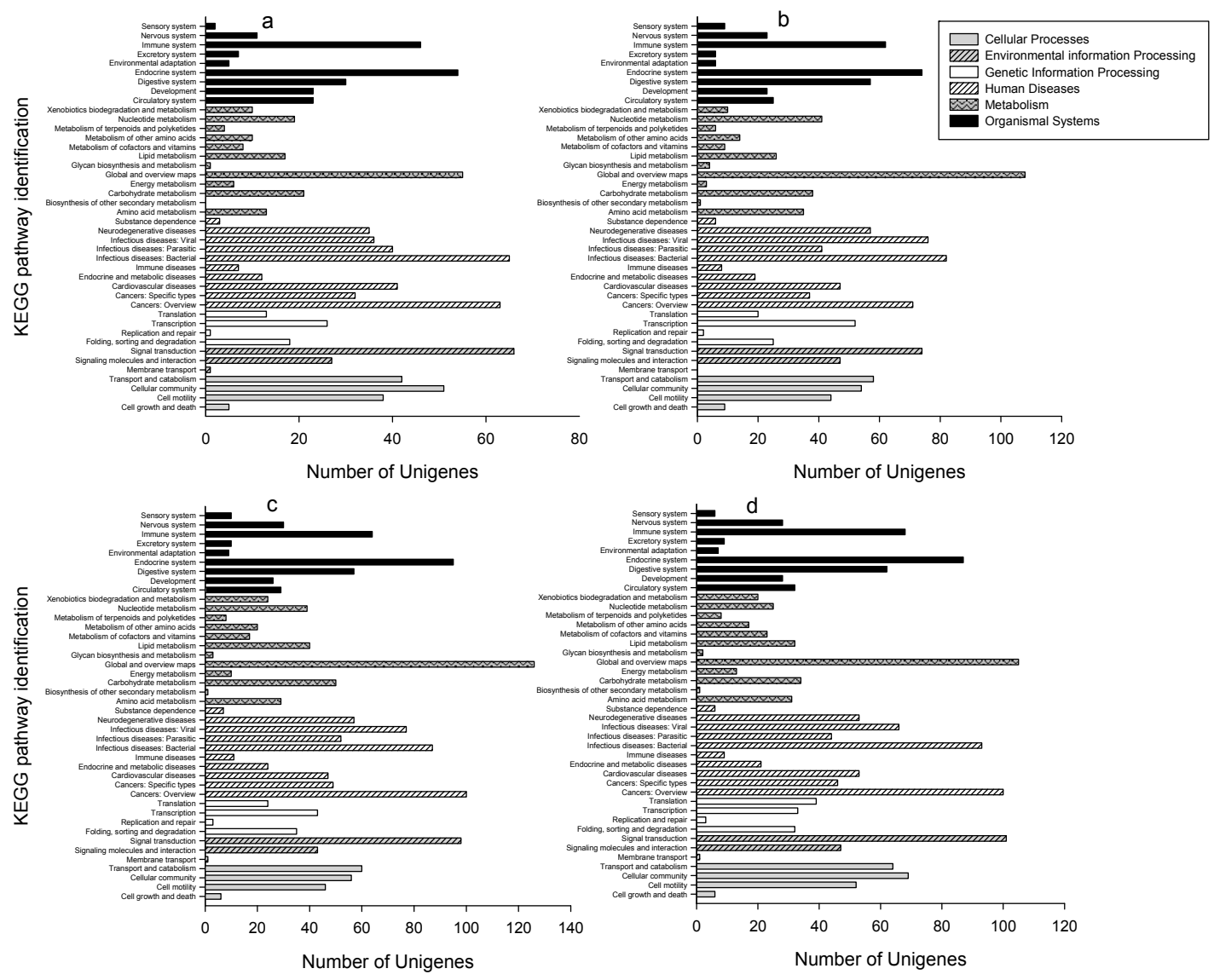

Figure 5. Number of DEUs assigned to KEGG pathway enrichment using different insecticides and different time durations in comparison with control. (a) treatment with chlorpyrifos for $6 \mathrm{~h}$ versus control, (b) treatment with chlorpyrifos for $48 \mathrm{~h}$ versus control, (c) treatment with clothianidin for $6 \mathrm{~h}$ versus control, (d) treatment with clothianidin for $48 \mathrm{~h}$ versus control.

Table 2. Most significant DEUs in control samples vs. those exposed to chlorpyrifos for $48 \mathrm{~h}$.

\begin{tabular}{|c|c|c|c|c|}
\hline Unigene ID & Annotation & $\begin{array}{l}\text { Expression Compared } \\
\text { to Control }\end{array}$ & Fold Change & KEGG Annotation \\
\hline CL2310.Contig3 & malate synthase A & Up & 14.06 & K01638 \\
\hline CL546.Contig2 & glycine-rich cell wall structural protein & $\mathrm{Up}$ & 14.06 & K10385 \\
\hline Unigene21344 & spliceosome-associated protein & $\mathrm{Up}$ & 13.96 & K13806 \\
\hline CL2019.Contig2 & keratin, type II cytoskeletal & $\mathrm{Up}$ & 13.37 & K11422 \\
\hline Unigene14906 & gastrointestinal growth factor & $\mathrm{Up}$ & 12.78 & - \\
\hline CL2724.Contig1 & cuticle protein & $\mathrm{Up}$ & 12.70 & K03006 \\
\hline Unigene15206 & cuticle protein & $\mathrm{Up}$ & 12.46 & K03006 \\
\hline CL1023.Contig2 & Lysosomal protein & $\mathrm{Up}$ & 12.13 & - \\
\hline Unigene 11830 & glycine-rich cell wall structural protein & $\mathrm{Up}$ & 11.97 & K13098 \\
\hline CL2019.Contig1 & keratin, type II cytoskeletal & $\mathrm{Up}$ & 11.92 & K07605 \\
\hline Unigene22580 & acyl-CoA desaturase & Down & -6.93 & K00507 \\
\hline Unigene21624 & endocuticle structural glycoprotein & Down & -8.62 & - \\
\hline Unigene 343 & cell target antigen & Down & -9.15 & - \\
\hline CL534.Contig2 & activating signal cointegrator & Down & -9.17 & K13114 \\
\hline CL1382.Contig3 & adhesion G-protein coupled receptor & Down & -9.44 & - \\
\hline Unigene1839 & Full=Flexible cuticle protein & Down & -9.92 & - \\
\hline CL2888.Contig2 & aldo-keto reductase protein & Down & -10.00 & K00011 \\
\hline CL572.Contig1 & $\mathrm{BTB} / \mathrm{POZ}$ domain-containing protein & Down & -10.32 & K10457 \\
\hline CL347.Contig2 & $\begin{array}{l}\text { Succinyl-CoA ligase (ADP/GDP-forming) } \\
\text { subunit } \alpha \text {, mitochondrial }\end{array}$ & Down & -10.63 & - \\
\hline Unigene6098 & Zonadhesin & Down & -14.62 & K07604 \\
\hline
\end{tabular}


Table 3. Most significant DEUs in control samples vs. those exposed to clothianidin for $48 \mathrm{~h}$.

\begin{tabular}{|c|c|c|c|c|}
\hline Unigene ID & Annotation & $\begin{array}{c}\text { Expression Compared to } \\
\text { Control }\end{array}$ & Fold Change & KEGG Annotation \\
\hline CL2903.Contig2 & Titin & Up & 11.58 & K14721 \\
\hline Unigene14493 & zinc finger protein & Up & 10.16 & K09228 \\
\hline CL2635.Contig1 & serine/threonine-protein kinase & Up & 9.78 & K08791 \\
\hline Unigene3337 & amino acid adenylation domain-containing protein & $\mathrm{Up}$ & 9.72 & K00142 \\
\hline Unigene5847 & tyrosine-protein phosphatase & Up & 9.52 & K18040 \\
\hline CL2809.Contig2 & transcription initiation factor & Up & 9.38 & K14650 \\
\hline Unigene2261 & histone acetyltransferase & $\mathrm{Up}$ & 9.25 & K11306 \\
\hline CL2708.Contig1 & aldose reductase & Down & -7.81 & K00011 \\
\hline Unigene26463 & dienelactone hydrolase family protein & Down & -8.96 & K01061 \\
\hline Unigene21654 & heat shock protein 67 & Down & -9.12 & K01011 \\
\hline Unigene20053 & keratin, type I cytoskeletal & Down & -9.49 & K07604 \\
\hline CL2528.Contig3 & cytochrome P450 & Down & -14.62 & K14999 \\
\hline
\end{tabular}

\subsection{Insecticide-Metabolism-Related DEUs and Quantitative Real-Time PCR Validation}

An analysis of the expression changes of insecticide-metabolism-related unigenes, such as carboxylesterase [16], catalase [17], cytochrome P450 [18], GST [19], nicotinamide adenine dinucleotide (NADH) dehydrogenase [20], trypsin [21], superoxide dismutase [22], acetylcholinesterase [23], gamma-aminobutyric acid (GABA) receptor [24], nicotinic acetylcholine receptor [25], and sodium channel $[26,27]$ after insecticide treatment is given in Table 4 . More than 50 P450-like unigenes were found to be expression-changed in all insecticide-treated samples in comparison with control, and the group of DEUs related to the P450-like unigenes was the largest group of DEUs related to insecticide metabolism (Table 4). Most GST-like unigenes were down-regulated after insecticide treatment, while only the same one GST-like unigene was up-regulated in the chlorpyrifos-treated sample for 6 and $48 \mathrm{~h}$, and the clothianidin-treated sample for $6 \mathrm{~h}$. For clothianidin-treated samples for $48 \mathrm{~h}$, all DEUs related to GST were down-regulated. The DEUs related to the GABA receptor showed consistency after insecticide treatment where all the three DEUs related to the GABA receptor were the same and were down-regulated. The same four DEUs related to the nicotinic acetylcholine receptor were found after treating the larvae with either chlorpyrifos or clothianidin for 6 or $48 \mathrm{~h}$; two of which were up-regulated and two down-regulated. Same eight DEUs related to the sodium channel were up-regulated when treated with chlorpyrifos for 6 and $48 \mathrm{~h}$, whereas in the case of clothianidin-treated larvae for 6 and $48 \mathrm{~h}$, only one out of eight DEUs related to the sodium channel was down-regulated, with seven up-regulated. Fourteen unigenes, including five P450-like unigenes, five GST-like unigenes, two carboxylesterase-like unigenes, and two cuticle-protein-like unigenes were used for the qRT-PCR to confirm the relative expression in different treatments. Figure 6 shows the results after comparing the qRT-PCR and FPKM value. Most of the chosen unigenes showed consistency of expression, however, seven out of 56 contrasts did not agree well. 

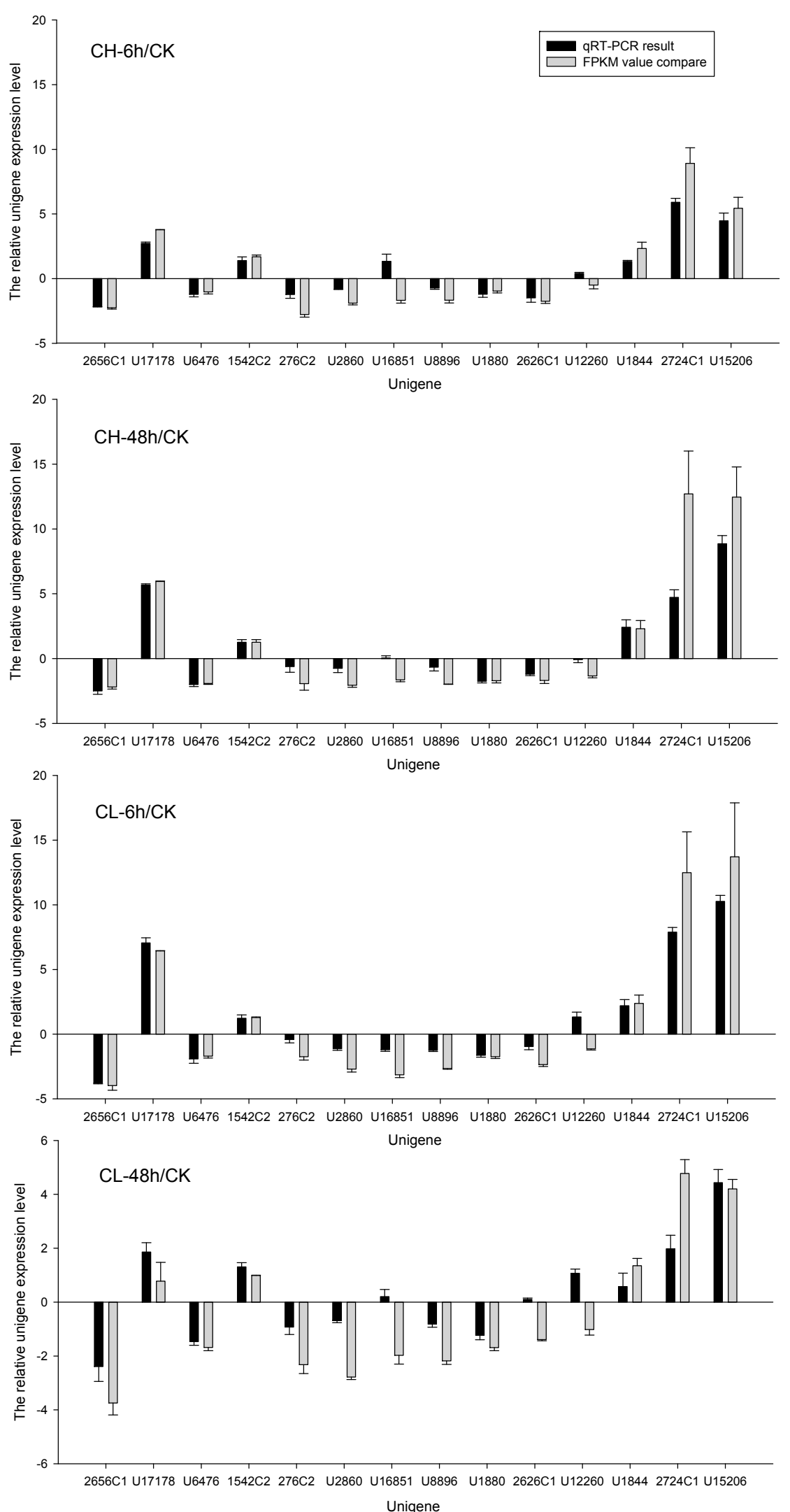

Figure 6. Insecticide-metabolism-related unigenes in comparison with control as confirmed by Quantitative Real-Time Polymerase Chain Reaction (qRT-PCR) and the calculated fragments per kilobase of transcript per million mapped reads (FPKM) values. CK: Control; CH: Chlorpyrifos; CL: Clothianidin; $6 \mathrm{~h}$ : treated $6 \mathrm{~h} ; 48 \mathrm{~h}$ : treated $48 \mathrm{~h}$. In the relative gene expression level (log2R) on $Y$-axis, $\mathrm{R}$ indicates the ratio of unigene expression in insecticides treated as compared with that in control. 
Table 4. DEUs related to insecticide metabolism. Up: Up-regulated expression; Down: Down-regulated expression. Up- or down-regulation indicates that the unigene's FPKM value for insecticide-treated samples is two times more or 0.5 times less than that in control.

\begin{tabular}{ccccccccc}
\hline \multirow{2}{*}{ Gene Name } & \multicolumn{2}{c}{ CH6h VS. CK } & \multicolumn{2}{c}{ CH48h VS. CK } & CL6h VS. CK & \multicolumn{2}{c}{ CL48h VS. CK } \\
\cline { 2 - 9 } & Up & Down & Up & Down & Up & Down & Up & Down \\
\hline Cytochrome P450 & 30 & 23 & 35 & 18 & 35 & 35 & 21 & 40 \\
Glutathione S-transferase & 1 & 31 & 1 & 29 & 1 & 37 & 0 & 35 \\
Carboxylesterase & 1 & 2 & 7 & 14 & 4 & 16 & 2 & 13 \\
Acetylcholinesterase & 5 & 2 & 5 & 6 & 7 & 5 & 4 & 6 \\
NADH dehydrogenase & 1 & 11 & 2 & 7 & 2 & 13 & 2 & 11 \\
Catalase & 3 & 1 & 4 & 1 & 2 & 1 & 3 & 1 \\
Trypsin & 10 & 14 & 20 & 34 & 18 & 18 & 8 & 38 \\
Superoxide dismutase & 3 & 2 & 1 & 1 & 1 & 4 & 2 & 0 \\
GABA receptor & 0 & 3 & 0 & 3 & 0 & 3 & 0 & 3 \\
Nicotinic acetylcholine receptor & 2 & 2 & 2 & 2 & 2 & 2 & 0 & 3 \\
Sodium channel & 8 & 0 & 8 & 0 & 7 & 1 & 7 & 1 \\
\hline
\end{tabular}

CK: Control; CH: Chlorpyrifos; CL: Clothianidin; 6 h: treated 6 h; 48 h: treated 48 h.

\section{Discussion}

Understanding of the unigenes involved in insect detoxification can provide new ideas for pest management, but insecticide metabolism is a complex biological event and is related to insecticide target proteins, and other cellular processes [28]. Next-generation sequencing technologies can promote research into insecticide detoxification in insects with unknown genomes or incomplete protein databases and help us to obtain more comprehensive knowledge of insecticide detoxification mechanisms. The underlying mechanism that involves the inhibition of acetylcholinesterase (AChE) is considered the major molecular mechanism of organophosphates against insects [29], whereas neonicotinoids kill the insects by continuous stimulation of nervous system through nicotinic acetylcholine receptors (nAChRs). Previous studies have mainly focused on these two mechanisms and the genes related to them. One concern that needs to be addressed, however, is to find out other gene/s that could be involved in the process of insect killing by chlorpyrifos and clothianidin. In this study, we found many DEUs after analyzing insecticide-treated samples versus control. Those DEUs were assigned to GO functional classification and KEGG pathway analysis.

For the Illumina HiSeq platform, the clean reads ranged between $90 \%$ and $99 \%$, and clean nucleotides Q20 usually around $97.0 \%$ [7,30,31]. In this study, the average of clean reads and clean nucleotides, Q20, was $99.89 \%$ and $98.01 \%$ respectively. These data show that BGISEQ-500 is a reliable platform for next generation sequencing. The clean reads mapped to the library sequence in the present study $(80.97 \%$ to $86.17 \%$, with an average of $83.56 \%$ for 15 samples) was a little higher than that of Xie [32], which ranged from $78.35 \%$ to $82.18 \%$, and lower than that of Ma [30], which ranged from $90.46 \%$ to $93.25 \%$, but almost the same as that of the Gao [8], who used the same species obtaining clean reads ranging between $79.91 \%$ and $85.76 \%$.

In the current study, the number of DEUs related to insecticide metabolism using the same insecticide but for different durations, was not much different, which shows that most insecticide-metabolism-related unigenes respond in $6 \mathrm{~h}$ (Figure 2). The DEUs of up-regulated and down-regulated patterns is almost the same for the intersection when treated with the same insecticide for different time durations (Figure 2a,c). However, for union, there were still some unigenes whose expression was not uniform (Figure $2 b, d$ ). The results of the cluster tree showed that the samples treated for either 6 or $48 \mathrm{~h}$ were clustered separately. This may indicate that the treatment duration of insecticide has more impact on the expression of unigenes than the type of insecticide (Figure 3).

The number of DEUs could be assigned to a GO functional classification and a GO functional group number, both of which were much smaller in the insecticide versus insecticide-treated samples, than in insecticide versus control. The same situation was noted for the DEUs mapped onto the KEGG pathways (Tables S2 and S4). The percentages of DEUs assigned to the three main GO categories 
were more stable in the insecticide versus control samples than in the insecticide versus insecticide samples (Table S3). This may indicate that the presence or absence of insecticide is a major factor for the DEUs of the insecticide-treated versus control treatment, which could be assigned into GO categories. However, comparing the insecticide-treated samples to each other does not seem to have much impact. Nevertheless, the duration of the insect exposure to these insecticides may determine their assignment to GO categories. In the significant DEUs of $48 \mathrm{~h}$ exposure to chlorpyrifos vs. control treatment, two of the 10 most up-regulated DEUs were annotated as a cuticle protein (Table 2). Cuticle proteins are associated with the regulation of molting and metamorphosis in insects [33]. In mosquitoes, insecticide resistance is also attributed to the chitinized cuticle, because it may impede the absorbance of the insecticide $[34,35]$. The cuticle protein genes differentially express as deltamethrin-susceptible and deltamethrin-resistant strains of Culex pipiens pallens [36], and are considered to have a role for resistance to deltamethrin in C. pipiens pallens [37]. Our results indicate that cuticle proteins may have a role in the detoxification of pesticides as well. In the most down-regulated unigenes for chlorpyrifos and clothianidin, both have one unigene mapped into the KEGG pathway, numbered K00011 and K07604 (Tables 2 and 3). In the present study, Lysosomal protein-like unigene (CL1023.Contig2) was listed in the 10 most up-regulated DEUs after $48 \mathrm{~h}$ exposure to chlorpyrifos. This protein has proved to be involved in the resistance of Musca domestica to organophosphorus insecticides [38]. Heat shock proteins (HSPs) are a family of proteins that are produced in response to stressful conditions, and their expression changes under the stress of insecticides [39]. Our results also show one HSP67 in the most down-regulated DEUs after $48 \mathrm{~h}$ exposure to clothianidin. Two unigenes mapped onto K00011 were annotated as aldo-keto reductase protein and aldose reductase protein in the NCBI Nucleotide database (Tables 2 and 3). Reductase protein plays an important role in insecticide detoxification metabolism, such as NADPH-Cytochrome P450 Reductase $[40,41]$. The most significant DEUs showed differences after treatment with clothianidin and chlorpyrifos, which may be due to the different modes of action of these insecticides, possible different impacts on off-target molecules or cellular/physiological pathways, or differences in how these insecticides may be metabolized based on their different structures.

Increasing the activity of the insecticide detoxification enzyme can help insects to survive insecticide exposure. Many enzyme families, such as P450, GST, and carboxylesterases are considered to be involved in insecticide detoxification mechanisms [42]. Cytochrome P450 monooxygenase-mediated metabolism is a common mechanism of insect detoxification to organophosphorus and neonicotinoid insecticides [43]. In each treatment, more than 50 DEUs were related to $\mathrm{P} 450$, which is considered the largest group of insecticide-metabolism-related unigenes. Also, it occupies a large proportion of all $\mathrm{P} 450$ genes, because insect genomes probably carry about 100 P450 genes [43]. GST is another large group of insecticide-related-unigenes, the number of which in the insect genomes is variable [44]. For example, Apis mellifera only contains eight GST genes [45], but Culex quinquefasciatus contains 38 GST genes [46]. In our study, each treatment had more than 30 DEUs related to GST, suggesting that almost all GSTs responded to insecticide treatment. Esterases, which include a large group of enzymes and which are considered as a mediated metabolic resistance of insecticides, have been detected in almost all insects [17]. The number of DEUs related to carboxylesterase in the chlorpyrifos-treated samples for $48 \mathrm{~h}$, was greater than those treated with chlorpyrifos for $6 \mathrm{~h}$, which may indicate that carboxylesterase-related unigenes respond more slowly than other insect-metabolism-related unigenes. This may provide an explanation as to why in some insects (brown plant hopper, mosquitoes, and aphids) the amplification of gene copies of carboxylesterase are shown in resistant strains, because more gene copies could help compensate for a slow transcriptional response [47-49]. Also, in some insects (mosquitoes, flies), mutations in carboxylesterase genes occur, showing enhanced detoxification of insecticide, perhaps to also compensate for the slow transcription of this family of detoxification proteins [50-52]. Most NADH dehydrogenase-related DEUs were down-regulated in each treatment, but Zhang et al. [53] report that the NADH dehydrogenase subunit six was up-regulated after the Allyl isothiocyanate fumigation, 
regardless of the concentration or fumigation time. This may be caused by the different insecticides used or different methods of insecticide application. In the current study, however, we fed the chive stem treated with insecticide to the insect larva, whereas Zhang et al. used fumigation. NADH dehydrogenase is the largest respiratory complex and the first enzyme of the mitochondrial electron transport chain. NADH dehydrogenase may behave differently and can have a different response mechanism to the way insecticides are applied, such as fumigation or feeding. In this study, we found many DEUs in B. odoriphaga after treating them with chlorpyrifos and clothianidin for 6 and $48 \mathrm{~h}$. Analysis of these DEUs can expand our understanding of resistance and detoxification mechanisms. Also, in future study, the DEUs can be treated as target genes for the detoxification mechanisms of $B$. odoriphaga. The specific genes involved in detoxification could be considered as molecular targets to explore novel approaches to control B. odoriphaga and to save the production of Chinese chive.

\section{Materials and Methods}

\subsection{Insect Rearing and Insecticide Treatment}

The initial culture of Chinese chive maggot (Bradysia odoriphaga) was collected from the bulbs

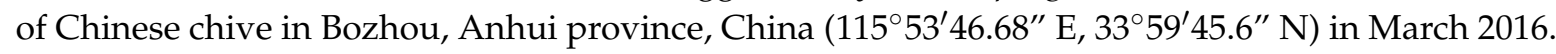
The maggot was then maintained in the laboratory on the stem of Chinese chives. In order to keep the Petri dish moistened, $1.5 \%$ agarose gel was heated and poured into the Petri dish to make a layer at the bottom of Petri dish. After the gel was solidified, a filter paper was placed on top of the gel. Chive stem was cut into 1-2 cm pieces and transferred to a plastic bowl with a cover on top. Several pairs of adults were then introduced into the bowl using an aspirator and covered with a lid. The adults laid eggs on the chive stems, which were then transferred to the Petri dish prepared before. After 7-10 days, the newly hatched larvae were shifted onto a fresh food. The food was refreshed after every 3-4 days until the larvae pupated. The rearing conditions were controlled by a walk-in climate chamber maintained at $25 \pm 1^{\circ} \mathrm{C}$, a photoperiod of $14 \mathrm{~h}$ light: $10 \mathrm{~h}$ darkness and $70 \pm 10 \%$ relative humidity [54]. Eggs, second instar larvae, fourth instar larvae, pupae and adults were collected for de novo transcriptome sequencing as a reference database. One milliliter insecticide solutions of chlorpyrifos (widely used for the control of Chinese chive maggots in Anhui province) and clothianidin (has not been used against chive maggot before in Anhui province) @ 25.1 and $0.5 \mu \mathrm{g} / \mathrm{L}$ respectively, (concentration was based on the LC50 value calculated after $48 \mathrm{~h}$ exposure) were added onto filter paper in the Petri dishes, which were used for rearing the fourth instar larvae of $B$. odoriphaga. The chive stems were plunged into the insecticide solution for $5 \mathrm{~s}$, and then fed to the fourth instar larvae for either 6 or $48 \mathrm{~h}$. Fourth instar larvae of $B$. odoriphaga feeding on chive stem (plunged in water for $5 \mathrm{~s}$ ) were considered as a control. Each treatment was replicated three times and the live larvae post-treatment were collected for RNA extraction and sequencing.

\subsection{RNA Extraction and cDNA Library Preparation}

Total RNA was extracted according to the manufacturer's protocol of an RNA isolation system (SV Total RNA Isolation System Kit, Promega Corporation, Madison, WI, USA). From the extracted RNA samples, the possible residual genomic DNA was removed by treating it with deoxyribonuclease (Dnase I: Fermentas Inc., Burlington, ON, Canada). RNA quality was determined using Agilent 2100 (Agilent Technologies, Santa Clara, CA, USA). The RIN (RNA integrated number) values of the sample chosen for RNA-Seq were between 6.4 and 7.2 (Table S5). The chosen RNA samples were treated as per standard procedure. Briefly, Oligo (dT) magnetic beads were used to select mRNA with a polyA tail and the target RNA obtained after purification. The target RNA was fragmented and reverse-transcribed to double-stranded cDNA (dscDNA) by N6 random primer. The dscDNA fragments were first end repaired with phosphate at $5^{\prime}$ end and stickiness " $A$ " at $3^{\prime}$ end, then adapters were ligated to the dscDNA fragments. PCR was performed to amplify the ligation product. The PCR 
product was then denatured by heat and the single strand DNA was cyclized by splint oligo and DNA ligase. Finally, the prepared library was sequenced by BGISEQ-500 sequencing platform.

\subsection{RNA-Seq Sequencing and DEU Screening}

Since no genomic sequence in any database was available for $B$. odoriphaga, we used a unigene database of $B$. odoriphaga transcriptome for all stages as a library sequence. The reference unigenes database was sequenced by Illumina HiSeq 2500 platform (Illumina, San Diego, CA, USA). The raw reads were deposited in the NCBI Sequence Read Archive database under the accession number SRP107066. The de novo assembly, sequence clustering, and functional categorization were done using the same method as that of Chen et al. [7]. Insecticide-treated samples were sequenced by BGISEQ-500 sequencing platform at the Beijing Genomics Institute (BGI; Shenzhen, China) and the raw reads were deposited in the NCBI Sequence Read Archive database under the accession number SRR5512549-SRR5512563. Clean reads were obtained after removing the reads with adaptors, reads having unknown nucleotides more than $5 \%$, and reads having more than $50 \%$ bases with Q-value $\leq 20$. The software Bowtie 2 was used to map the clean reads to assembled reference sequence database and then the fragment for each unigene was counted from the mapping results [55]. The relative expression of the unigenes was calculated by dividing the unigene's FPKM value in insecticide-treated samples with the same unigene FPKM value in control. We then identified the DEUs with an absolute value of $\log _{2}$ Ratio $>1$ or $\log _{2}$ Ratio $<-1$. The formula for FPKM value is as follows:

Where $C$ is the number of fragments that is uniquely aligned to the calculated unigene, $N$ is the total number of fragments that is uniquely aligned to all unigenes and $L$ is the base number of the calculated unigene.

\subsection{DEUs, GO Annotation and Pathway Enrichment among Different Treatments}

GO is an international standard in functional classification systems. Blast2go PRO software was used for analyzing DEUs assigned to a GO functional group [56], and unigenes with annotation were distributed into three main ontologies: molecular function, cellular component, and biological process [57]. Pathway analysis helps to further understand the DEUs' biological functions. The detected DEUs were also annotated against the Kyoto Encyclopedia of Genes and Genomes (KEGG) database [58].

\subsection{Sequence Confirmation and Quantitative Real-Time Polymerase Chain Reaction ( $q$ RT-PCR) Validation}

Thirteen expression-changed unigene sequences including five CytochromesP450 (P450) genes, five glutathione S-transferase (GST) genes, two AChE genes, and one reference gene glyceraldehyde-3-phosphate dehydrogenase (GAPDH), were confirmed by qRT-PCR. Specific primers were designed using Primer premier 5.0, and then reverse transcription PCR was conducted. After electrophoresis of the PCR products, the DNA band was extracted by Agarose Gel Extraction kit (Takara Biotechnology (Dalian) Co., Ltd., Dalian, China), and then subcloned into vector (pEASY-T1 Simple Cloning Kit, Beijing TransGen Biotech Co., Ltd., Beijing, China) according to the manufacturer's protocol. A 3730 DNA Analyzer was used to determine the nucleotide sequences. The confirmed sequences were deposited in the GenBank database with the accession numbers KY997061-KY997073, and MG407793-MG407796.

The relative expression of 14 confirmed sequences was determined by qRT-PCR, choosing the GAPDH as the reference gene. Beacon Designer 7.0 was used to design the primers for qRT-PCR, and one cycle amplification efficiency of the primers was calculated [59]. cDNA was synthesized from one microgram of total RNA after removing the genomic DNA (PrimeScript ${ }^{\mathrm{TM}}$ RT Reagent Kit with gDNA Eraser, Takara Biotechnology (Dalian) Co., Ltd.). Cycle threshold (Ct) value of GAPDH in each cDNA sample was used to adjust the concentrations of cDNA [60]. The qRT-PCR was performed on a CFX96 Real-Time PCR Detection System (Bio-Rad Laboratories, Inc., Hercules, CA, USA) using SYBR Premix (Takara Biotechnology (Dalian) Co., Ltd.) according to the manufacturer's instructions. The thermal 
cycling conditions were: 45 cycles at $95^{\circ} \mathrm{C}$ for $30 \mathrm{~s}, 56{ }^{\circ} \mathrm{C}$ for $20 \mathrm{~s}$, and $72{ }^{\circ} \mathrm{C}$ for $30 \mathrm{~s}$. There were four biological samples and each had two technical replicates to determine the relative expression of unigenes. The delta $\mathrm{Ct}$ value of the target gene and reference gene for each sample was first calculated, and then the relative expression to the reference gene was determined by using the delta $\mathrm{Ct}$ value and the efficiency of the primer. The relative expression of each gene in pesticide-treated samples vs. control was calculated. The NCBI accession number, primers designed for confirmation and qRT-PCR are listed in Table S6. The PCR amplification efficiencies of primers for qRT-PCR are shown in Table S7.

\section{Conclusions}

This study provides information about the genes of B. odoriphaga involved in responding to two insecticides, chlorpyrifos and clothianidin. This information can expand our understanding of resistance and detoxification mechanisms of this insect. The study further provides analysis of the most significantly up- and down-regulated DEUs and insecticide metabolism related DEUs. These genes could be further studied and used as molecular targets to explore novel approach to control B. odoriphaga.

Supplementary Materials: Supplementary materials can be found at www.mdpi.com/1422-0067/18/11/2445/s1.

Acknowledgments: This study was supported by the National Natural Science Funds of China (31401746), the Chinese Special Fund for Agro-scientific Research in the Public Interest (201303027) and Public Welfare Technology Application Research of Anhui Province (1704f0704073).

Author Contributions: Haoliang Chen and Weihua Su conceived and designed the experiments. Lulu Lin and Minghui Xie performed the experiments. Haoliang Chen and Guangling Zhang analyzed the data. Haoliang Chen and Farman Ali interpreted the results and wrote the paper.

Conflicts of Interest: The authors declare no conflict of interest.

\section{References}

1. Mei, Z.; Wu, Q.; Zhang, Y.; Hua, L. The biology, ecology and management of Bradysia odoriphaga. Entomol. Knowl. 2003, 40, 396-398.

2. Zhou, X.; Cao, X.; Shen, Y.; Zhuang, Q.; Zhang, S.; Yu, Y.; Zhang, A. Effects of supplementary nutrition and water on the reproduction and longevity of Bradysia odoriphaga. Chin. J. Appl. Entomol. 2016, 53, 1205-1210.

3. Dang, Z.; Dong, J.; Gao, Z.; Jia, H.; Zhang, K.; Pan, W. Biology and injury of Bradysia odoriphaga on leek in different types of cultivation. J. Agric. Univ. Hebei 2001, 24, 65-68.

4. Zhang, Z.; Zhang, Z.; Li, W.; He, M.; Zhu, X.; Lin, W.; Li, X.; Li, W.; Zhang, J.; Bei, Y.; et al. Behavioral responses of female Bradysia odoriphaga Yang et Zhang to volatiles of conspecific larvae, pupae and eggs. Chin. J. Appl. Entomol. 2016, 53, 1198-1204.

5. Song, J.; Cao, W.; Feng, S.; Du, L. Effectiveness of high virulence Bt resources as a means of controlling Bradysia odoriphaga larvae. Chin. J. Appl. Entomol. 2016, 53, 1217-1224.

6. Ffrenchconstant, R.H. The molecular genetics of insecticide resistance. Genetics 2013, 194, 807-815. [CrossRef] [PubMed]

7. Chen, H.; Lin, L.; Xie, M.; Zhang, G.; Su, W. De novo sequencing and characterization of the Bradysia odoriphaga (Diptera: Sciaridae) larval transcriptome. Comp. Biochem. Phys. D 2015, 16, 20-27. [CrossRef] [PubMed]

8. Gao, H.; Zhai, Y.; Wang, W.; Chen, H.; Zhou, X.; Zhuang, Q.; Yu, Y.; Li, R. Transcriptome analysis and discovery of genes relevant to development in Bradysia odoriphaga at three developmental stages. PLoS ONE 2016, 11, e0146812. [CrossRef] [PubMed]

9. Schuster, S.C. Next-generation sequencing transforms today's biology. Nat. Methods 2008, 5, 16-18. [CrossRef] [PubMed]

10. Zhang, J.; Chiodini, R.; Badr, A.; Zhang, G. The impact of next-generation sequencing on genomics. J. Genet. Genom. 2011, 38, 95-109. [CrossRef] [PubMed]

11. Van Nimwegen, K.; Soest, R.; Veltman, J.; Nelen, M.; Wilt, G.; Vissers, L.; Grutters, J. Is the $\$ 1000$ Genome as Near as We Think? A Cost Analysis of Next-Generation Sequencing. Clin. Chem. 2016, 62, 1458-1464. [CrossRef] [PubMed] 
12. Mamidala, P.; Wijeratne, A.; Wijeratne, S.; Kornacker, K.; Sudhamalla, B.; Rivera-Vega, L.; Hoelmer, A.; Meulia, T.; Jones, S.; Mittapalli, O. RNA-Seq and molecular docking reveal multi-level pesticide resistance in the bed bug. BMC Genom. 2012, 13, 6. [CrossRef] [PubMed]

13. Lei, Y.; Zhu, X.; Xie, W.; Wu, Q.; Wang, S.; Guo, Z.; Xu, B.; Li, X.; Zhou, X.; Zhang, Y. Midgut transcriptome response to a Cry toxin in the diamondback moth, Plutella xylostella (Lepidoptera: Plutellidae). Gene 2014, 533, 180-187. [CrossRef] [PubMed]

14. Bajda, S.; Dermauw, W.; Greenhalgh, R.; Nauen, R.; Tirry, L.; Clark, R.; Van Leeuwen, T. Transcriptome profiling of a spirodiclofen susceptible and resistant strain of the European red mite Panonychus ulmi using strand-specific RNA-seq. BMC Genom. 2015, 16, 974. [CrossRef] [PubMed]

15. Liu, X.; Wu, D.; Zhang, Y.; Zhou, H.; Lai, T.; Ding, W. RNA-Seq Analysis Reveals Candidate Targets for Curcumin against Tetranychus cinnabarinus. Biomed. Res. Int. 2016, 2016, 2796260. [PubMed]

16. Cui, F.; Li, M.; Chang, H.; Mao, Y.; Zhang, H.; Lu, L.; Yan, S.; Lang, M.; Liu, L.; Qiao, C. Carboxylesterase-mediated insecticide resistance: Quantitative increase induces broader metabolic resistance than qualitative change. Pestic. Biochem. Physiol. 2015, 121, 88-96. [CrossRef] [PubMed]

17. Al-Ayedh, H.; Rizwan-ul-Haq, M.; Hussain, A.; Aljabr, A.M. Insecticidal potency of RNAi-based catalase knockdown in Rhynchophorus ferrugineus (Oliver) (Coleoptera: Curculionidae). Pest Manag. Sci. 2016, 72, 2118-2127. [CrossRef] [PubMed]

18. Wen, Z.; Zhang, X.; Zhang, Y. P450-Mediated Insecticide Detoxification and Its Implication in Insecticide Efficacy. In Recent Advances in Entomological Research; Liu, T., Kang, L., Eds.; Springer: Berlin, Germany, 2011; pp. 229-245.

19. Enayati, A.; Ranson, H.; Hemingway, J. Insect glutathione transferases and insecticide resistance. Insect Mol. Biol. 2005, 14, 3-8. [CrossRef] [PubMed]

20. Hollingworth, R.; Ahammadsahib, K. Inhibitors of respiratory complex I: Mechanisms, pesticidal actions and toxicology. Rev. Pestic. Toxicol. 1995, 3, 277-302.

21. Borovsky, D.; Rabindran, S.; Dawson, W.; Powell, C.; Iannotti, D.; Morris, T.; Shabanowitz, J.; Hunt, D.; DeBondt, H.; DeLoof, A. Expression of Aedes trypsin-modulating oostatic factor on the virion of TMV: A potential larvicide. Proc. Natl. Acad. Sci. USA 2006, 103, 18963-18968. [CrossRef] [PubMed]

22. Lomate, P.; Sangole, K.; Sunkar, R.; Hivrale, V. Superoxide dismutase activities in the midgut of Helicoverpa armigera larvae: Identification and biochemical properties of a manganese superoxide dismutase. Open Access Insect Physiol. 2015, 5, 13-20.

23. Menozzi, P.; An Shi, M.; Lougarre, A.; Tang, Z.; Fournier, D. Mutations of acetylcholinesterase which confer insecticide resistance in Drosophila melanogaster populations. BMC Evol. Biol. 2004, 4, 4. [CrossRef] [PubMed]

24. Ffrench-constant, R.; Rocheleau, T.; Steichen, J.; Chalmers, A. A point mutation in a Drosophila GABA receptor confers insecticide resistance. Nature 1993, 363, 449-451. [CrossRef] [PubMed]

25. Liu, Z.; Williamson, M.; Lansdell, S.; Denholm, I.; Han, Z.; Millar, N. A nicotinic acetylcholine receptor mutation conferring target-site resistance to imidacloprid in Nilaparvata lugens (brown planthopper). Proc. Natl. Acad. Sci. USA 2005, 102, 8420-8425. [CrossRef] [PubMed]

26. Bloomquist, J. Ion channels as targets for insecticides. Ann. Rev. Entomol. 1996, 41, 163-190. [CrossRef] [PubMed]

27. Zlotkin, E. The insect voltage-gated sodium channel as target of insecticides. Ann. Rev. Entomol. 1999, 44, 429-455. [CrossRef] [PubMed]

28. Perry, T.; Batterham, P.; Daborn, P. The biology of insecticidal activity and resistance. Insect Biochem. Mol. 2011, 41, 411-422. [CrossRef] [PubMed]

29. Eddleston, M.; Buckley, N.; Eyer, P.; Dawson, A. Management of acute organophosphorus pesticide poisoning. Lancet 2008, 371, 597-607. [CrossRef]

30. Ma, J.; Wang, R.; Li, X.; Gao, B.; Chen, S. Transcriptome and gene expression analysis of Cylas formicarius (Coleoptera: Brentidae) during different development stages. J. Insect Sci. 2016, 16, 63. [CrossRef] [PubMed]

31. Chen, H.; Lin, L.; Xie, M.; Zhang, G.; Su, W. De novo sequencing, assembly and characterization of antennal transcriptome of Anomala corpulenta Motschulsky (Coleoptera: Rutelidae). PLoS ONE 2014, 9, e114238. [CrossRef] [PubMed]

32. Xie, M.; Huang, Y.; Zhang, Y.; Wang, X.; Yang, H.; Yu, O.; Dai, W.; Fang, C. Transcriptome profiling of fruit development and maturation in Chinese white pear (Pyrus bretschneideri Rehd). BMC Genom. 2013, 14, 823. [CrossRef] [PubMed] 
33. Charles, J. The regulation of expression of insect cuticle protein genes. Insect Biochem. Mol. Biol. 2010, 40, 205-213. [CrossRef] [PubMed]

34. Ahmad, M.; McCaffery, A.R. Elucidation of detoxication mechanisms involved in resistance to insecticides in the third instar larvae of a field-selected strain of Helicoverpa armigera with the use of synergists. Pestic. Biochem. Physiol. 1991, 41, 41-52. [CrossRef]

35. Kostaropoulos, I.; Papadopoulos, A.I.; Metaxakis, A.; Boukouvala, E.; Papadopoulou-Mourkidou, E. Glutathione S-transferase in the defence against pyrethroids in insects. Insect Biochem. Mol. Biol. 2001, 31, 313-319. [CrossRef]

36. Wang, W.; Lv, Y.; Fang, F.; Hong, S.; Guo, Q.; Hu, S.; Zou, F.; Shi, L.; Lei, Z.; Ma, K.; et al. Identification of proteins associated with pyrethroid resistance by iTRAQ-based quantitative proteomic analysis in Culex pipiens pallens. Parasites Vectors 2015, 8, 95. [CrossRef] [PubMed]

37. Fang, F.; Wang, W.; Zhang, D.; Lv, Y.; Zhou, D.; Ma, L.; Shen, B.; Sun, Y.; Zhu, C. The cuticle proteins: A putative role for deltamethrin resistance in Culex pipiens pallens. Parasitol. Res. 2015, 114, 4421-4429. [CrossRef] [PubMed]

38. Wilkins, R.M.; Ahmed, S.; Mantle, D. Comparative effect of fenitrothion treatment on intracellular protease activities in insecticide-resistant and susceptible strains of Musca domestica L. Comp. Biochem. Phys. C 1999, 124, 337-343. [CrossRef]

39. Wang, H.; Reitz, S.R.; Wang, L.; Wang, S.; Li, X.; Lei, Z. The mRNA expression profiles of five heat shock protein genes from Frankliniella occidentalis at different stages and their responses to temperatures and insecticides. J. Integr. Agric. 2014, 13, 2196-2210. [CrossRef]

40. Zhu, F.; Sams, S.; Moural, T.; Haynes, K.; Potter, M.; Palli, S. RNA Interference of NADPH-Cytochrome P450 Reductase Results in Reduced Insecticide Resistance in the Bed Bug, Cimex lectularius. PLoS ONE 2012, 7, e31037. [CrossRef] [PubMed]

41. Zhang, Y.; Wang, Y.; Wang, L.; Yao, J.; Guo, H.; Fang, J. Knockdown of NADPH-cytochrome P450 reductase results in reduced resistance to buprofezin in the small brown planthopper, Laodelphax striatellus (fallén). Pestic. Biochem. Physiol. 2016, 127, 21-27. [CrossRef] [PubMed]

42. Li, X.; Schuler, M.; Berenbaum, M. Molecular mechanisms of metabolic resistance to synthetic and natural xenobiotics. Annu. Rev. Entomol. 2007, 52, 231-253. [CrossRef] [PubMed]

43. Feyereisen, R. Insect P450 enzymes. Annu. Rev. Entomol. 1999, 44, 507-533. [CrossRef] [PubMed]

44. Fang, S. Insect glutathione S-transferase: A review of comparative genomic studies and response to xenobiotics. Bull. Insectol. 2012, 65, 265-271.

45. Claudianos, C.; Ranson, H.; Johnson, R.; Biswas, S.; Schuler, M.; Berenbaum, M.; Feyereisen, R.; Oakeshott, J. A deficit of detoxification enzymes: Pesticide sensitivity and environmental response in the honeybee. Insect Mol. Biol. 2006, 15, 615-636. [CrossRef] [PubMed]

46. Friedman, R. Genomic organization of the glutathione S-transferase family in insects. Mol. Phylogenet. Evol. 2011, 61, 924-932. [CrossRef] [PubMed]

47. Small, G.J.; Hemingway, J. Molecular characterization of the amplified carboxylesterase gene associated with organophosphorus insecticide resistance in the brown planthopper, Nilaparvata lugens. Insect Mol. Biol. 2000, 9, 647-653. [CrossRef] [PubMed]

48. Hemingway, J.; Karunaratne, S.H. Mosquito carboxylesterases: A review of the molecular biology and biochemistry of a major insecticide resistance mechanism. Med. Vet. Entomol. 1998, 12, 1-12. [CrossRef] [PubMed]

49. Xi, J.; Pan, Y.; Bi, R.; Gao, X.; Chen, X.; Peng, T.; Zhang, M.; Zhang, H.; Hu, X.; Shang, Q. Elevated expression of esterase and cytochrome $\mathrm{P} 450$ are related with lambda-cyhalothrin resistance and lead to cross resistance in Aphis glycines Matsumura, Pest. Biochem. Physiol. 2015, 118, 77-81. [CrossRef] [PubMed]

50. Cui, F.; Lin, Z.; Wang, H.; Liu, S.; Chang, H.; Reeck, G.; Qiao, C.; Raymond, M.; Kang, L. Two single mutations commonly cause qualitative change of nonspecific carboxylesterases in insects. Insect Biochem. Mol. Biol. 2011, 41, 1-8. [CrossRef] [PubMed]

51. Newcomb, R.D.; Campbell, P.M.; Ollis, D.L.; Cheah, E.; Russell, R.J.; Oakeshott, J.G. A single amino acid substitution converts a carboxylesterase to an organophosphorus hydrolase and confers insecticide resistance on a blowfly. Proc. Natl. Acad. Sci. USA 1997, 94, 7464-7468. [CrossRef] [PubMed] 
52. Devonshire, A.L.; Heidari, R.; Bell, K.L.; Campbell, P.M.; Campbell, B.E.; Odgers, W.A.; Oakeshott, J.G.; Russell, R.J. Kinetic efficiency of mutant carboxylesterases implicated in organophosphate insecticide resistance. Pestic. Biochem. Physiol. 2003, 76, 1-13. [CrossRef]

53. Zhang, C.; Ma, Z.; Zhang, X.; Wu, H. Transcriptomic alterations in Sitophilus zeamais in response to allyl isothiocyanate fumigation. Pestic. Biochem. Physiol. 2017, 137, 62-70. [CrossRef] [PubMed]

54. Mei, Z.; Wu, Q.; Zhang, Y.; Hua, L. Life tables of the laboratory population of Bradysia odoriphaga Yang et Zhang (Diptera: Mycetophilidae) at different temperatures. Acta Entomol. Sin. 2004, 47, 219-222.

55. Langmead, B.; Trapnell, C.; Pop, M.; Salzberg, S. Ultrafast and memory-efficient alignment of short DNA sequences to the human genome. Genome Biol. 2009, 10, 25-34. [CrossRef] [PubMed]

56. Conesa, A.; Götz, S.; García-Gómez, J.; Terol, J.; Talón, M.; Robles, M. Blast2GO: A universal tool for annotation, visualization and analysis in functional genomics research. Bioinformatics 2005, 21, 3674-3676. [CrossRef] [PubMed]

57. Ashburner, M.; Ball, C.; Blake, J.; Botstein, D.; Butler, H.; Cherry, J.; Davis, A.; Dolinski, K.; Dwight, S.; Eppig, J.; et al. Gene Ontology: Tool for the unification of biology. Nat. Genet. 2000, 25, 25-29. [CrossRef] [PubMed]

58. Kanehisa, M.; Araki, M.; Goto, S.; Hattori, M.; Hirakawa, M.; Itoh, M.; Katayama, T.; Kawashima, S.; Okuda, S.; Tokimatsu, T.; et al. KEGG for linking genomes to life and the environment. Nucleic Acids Res. 2008, 36, 480-484. [CrossRef] [PubMed]

59. Pfaffl, M.W. A new mathematical model for relative quantification in real-time RT-PCR. Nucleic Acids Res. 2001, 29, e45. [CrossRef] [PubMed]

60. Zhu, F.; Xu, J.; Palli, R.; Ferguson, J.; Palli, S. Ingested RNA interference for managing the populations of the Colorado potato beetle, Leptinotarsa decemlineata. Pest Manag. Sci. 2011, 67, 175-182. [CrossRef] [PubMed]

(C) 2017 by the authors. Licensee MDPI, Basel, Switzerland. This article is an open access article distributed under the terms and conditions of the Creative Commons Attribution (CC BY) license (http:/ / creativecommons.org/licenses/by/4.0/). 